\title{
The Ouchi illusion: An anomaly in the perception of rigid motion for limited spatial frequencies and angles
}

\author{
TREVOR HINE, MICHAEL COOK, and GARRY T. ROGERS \\ Australian National University, Canberra, Australia
}

\begin{abstract}
The spatial parameters underlying a novel illusion of relative motion are characterized. A simple stimulus composed of two sine-wave gratings was sufficient to generate the illusion. We measured the response of subjects to rapid, small-amplitude oscillations of this stimulus behind a fixation point. The effect was clearly strongest for acute angles between the gratings, but only when spatial frequency was between 6 and $11 \mathrm{cpd}$. We surmise that activity in the grating cells of the primate visual cortex (von der Heydt, Peterhans, \& Dursteler, 1992) might be the cause of the illusion. The illusion is potentially an important tool in understanding how higher cortical areas combine disparate motion signals.
\end{abstract}

The cover illustration of a recent text (Spillmann \& Werner, 1990) has drawn attention to a design by the Japanese graphic artist Hajime Ouchi (Ouchi, 1977, p. 75) that generates a novel illusion of relative motion. Although this "Ouchi illusion" has aroused interest in many perception laboratories, we are aware of only two reports (Khang, He, \& Essock, 1995; Spillmann, Tulunay-Keesey, \& Olson, 1993) and one paper (Hine, Cook, \& Rogers, 1995) that have investigated the effect formally. A version of the Ouchi design is presented in Figure 1 and consists of a surround region filled with oblong checks and an inset in which the same texture has been rotated through $90^{\circ}$. When the pattern is viewed at an appropriate distance, illusory relative movement is frequently seen, comprising small, smooth displacements of the inset that may or may not be accompanied by movements of the surround in a different direction. The effect is seen by many observers as a motion in two depth planes, but it seems likely that the apparent depth difference between inset and surround is a secondary consequence of the appearance of motion in an aperture. The illusory relative motion can be enhanced by physically jiggling the pattern. With free viewing, the direction of relative motion is difficult to identify and is unstable. However, prolonged apparent movement in a specific direction can be produced by tracking a fixation point moving slowly across the pattern or, equivalently, by moving the pattern behind a stationary fixation point. By these means and following Spillmann et al.'s (1993) observation, readers can confirm for themselves that apparent movement in Figure 1 seems to be strongest when the fixation path is oblique.

G.T.R. is now with the Department of Psychology, University of Melbourne, Parkville, Victoria, Australia. Correspondence should be addressed to T. Hine, who is now at the School of Applied Psychology, Griffith University, QLD 4111, Australia (e-mail: t.hine@hbs.gu.edu.au).
These observations suggest strongly that the effect is due to movement of the pattern's image upon the retina, and this has been confirmed by Spillmann et al. (1993), who generated illusory movement by moving the Ouchi pattern within a stabilized retinal image. The movement evident during free viewing of the pattern is presumably due to the drifts or microsaccades that occur during fixation (Steinman, Haddad, Skavenski, \& Wyman, 1973). Although all parts of the pattern are moving en bloc in a given direction, it appears that the local motion directions of the center and surround are encoded differently.

We attempted to identify the critical spatial features of the Ouchi illusion by modifying the checkerboard pattern in various ways. Exploratory observations indicated that the effect is strongly size dependent. Thus, the motion in Figure 1 is evident only while the whole pattern subtends visual angles in the approximate range of $2^{\circ}$ to $9^{\circ}$. Other observations indicated that the effect is not dependent on juxtaposition of the inset and surround, for it occurs when the two regions are separated by a gap, as in Figure 1, or if the inset is simply flanked by patches containing the checkerboard texture. The effect is evident over a wide range of aspect ratios and sizes for the checks constituting the pattern.

The inset and surround in the Ouchi pattern can be differentiated in two ways. The first is spatial frequency. Along horizontal slices through the checkerboard patterns, the inset has higher spatial frequency than the surround, and this is reversed for vertical slices. It is possible that the differences in perceived motion as the pattern moves on the retina are a consequence of the local differences in spatiotemporal frequency in the center and surround. The second feature that obviously distinguishes the two regions of the pattern is the difference in the orientations of their respective oblique (1-D) harmonic components: In the inset, the highest contrast components approach vertical, whereas in the surround they approach horizontal. As the whole pattern moves vertically, for example, 


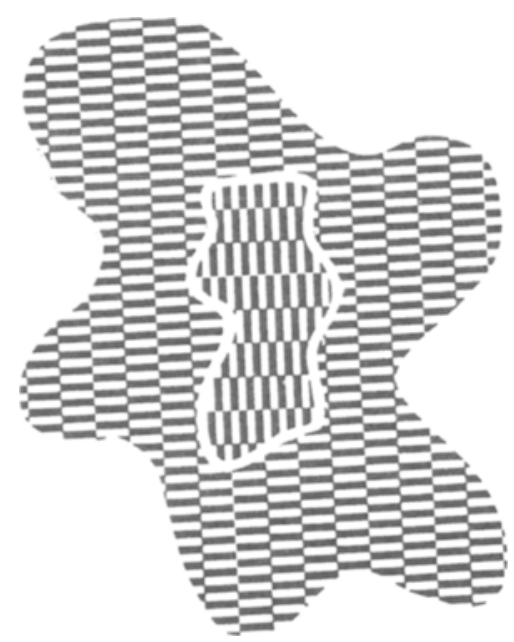

Figure 1. A stimulus of nonsymmetrical shape filled with a texture of oblong checks and based on Ouchi (1977) with the addition of a small gap between inset and surround. When viewed at reading distance, the inset will frequently seem to move with respect to the surrounding checkerboard, appearing either as if viewed through an aperture or as an occluding figure.

the local motions of these oblique harmonics will be in directions orthogonal to their orientations, and the inset and the surround directions will be different. This observation leads to the hypothesis that the motion in the Ouchi pattern arises because our perception is determined by the local motions of the harmonic components of its two regions, rather than the global motion of these regions, or, for that matter, the local motions of the rectangles constituting the checkerboards.

On the basis of this hypothesis, we anticipated that a similar pattern of illusory movement would be generated by replacing the inner and outer checkered regions of the Ouchi figure with gratings of contrasting orientations. The inset in our display (Figure 2, upper stimulus) consisted of a sinusoidal grating of a particular orientation and frequency. The surround composed a grating of the same frequency, but a different orientation. The reader may confirm by inspection of either stimuli in Figure 2 that this simplified pattern generates the illusion of relative motion. The occurrence of this effect in Figure 2, although not directly establishing our analysis of the Ouchi illusion, does provide support for it. Apparent relative motion was generated by oscillating this stimulus behind a stationary fixation point at a rate that precluded eye tracking. The simplified nature of the stimulus allowed us to directly assess the effect of variations of spatial frequency and orientation contrast on the incidence and strength of the illusory movement. Ancillary experiments examined the effects of variations in grating contrast and spatial frequencies on inset and surround.

\section{METHOD}

\section{Subjects}

Four subjects participated in the experiments, 2 of whom (T.J.H., G.T.R.) were authors and the other 2 of whom were naive to the purposes of the experiment. Three subjects possessed normal or corrected-to-normal vision, and all wore their corrections during the experiment. Subject L.V. was strongly amblyopic (suppressing the left eye) with some hypermetropia (LE: $+2 \mathrm{D}, \mathrm{RE}+0.5 \mathrm{D})$. She had undergone corrective surgery for strabismus as a young child.

\section{Apparatus and Stimuli}

The stimuli were displayed on a Tektronix 690SR color monitor controlled by a custom-built color graphics interface (PDI; James Sokoll Pty Ltd., Milton, QLD, Australia). Resolution of the display was $512 \times 512$ pixels refreshed at $60 \mathrm{~Hz}$ noninterlaced with red, green, and blue guns each driven by a 12-bit D/A converter. The monitor was free viewed binocularly in a front-silvered mirror within a light-tight tunnel situated in a darkened laboratory and was placed $210 \mathrm{~cm}$ from the observer's eyes. The mirror was used to deflect the viewing path away from another optical setup in the laboratory. Graphics and data collection were controlled by the laboratory minicomputer.

Figure 3 is a schematic of our stimulus. It was superimposed on a constant white (chromaticity: $x=0.33, y=0.34$ ) background, $5.85^{\circ}$ a side and with a luminance of $149 \mathrm{cdm}^{-2}$. The stimulus was
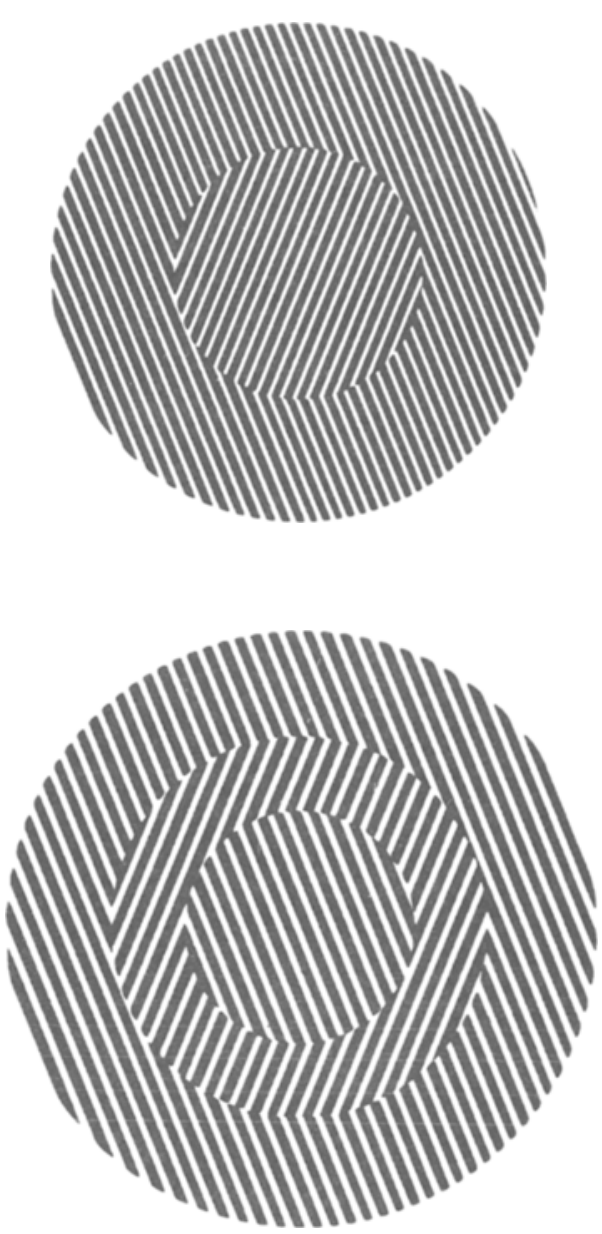

Figure 2. The upper stimulus is very similar to the stimulus used in the main experiment. When viewed at around $50 \mathrm{~cm}$ and gently jiggled up and down at ca. $3 \mathrm{~Hz}$, the inner disk seems to slide to and fro above the outer ring, or as if seen through a hole. The effect is not as pronounced if the figure is held sideways before being jiggled up and down. In the lower stimulus, it is the middle annulus that will be perceived to move most of the time. Further demonstrations are described in the text. 
annular, with the outer ring (surround) subtending $2.9^{\circ}$, which was twice the diameter of the inner disk (inset). Both the inset and surround were striped with 1-D sine-wave modulations of achromatic contrast of the same fixed spatial frequency, except in one experiment, described below, in which the spatial frequencies of the inset and surround were independently varied. The mean luminance of this waveform was the same as the background, with contrast $(C)$ defined as the Michelson contrast such that $C=\left(L_{\max }-L_{\min }\right) /\left(L_{\max }\right.$ $\left.+L_{\min }\right)$, where $L_{\max }$ is the peak luminance and $L_{\min }$ is the trough luminance. The surround and inset grating were tilted symmetrically about the vertical axis: $\theta / 2$ to the left and right, respectively. In each trial, the stimulus appeared on the screen and immediately oscillated vertically (triangular wave, amplitude $=0.13^{\circ}$, indicated by double-ended arrow in Figure 3) at $3.8 \mathrm{~Hz}$ for four cycles (velocity $=0.98^{\circ} / \mathrm{sec}$; duration $=1,070 \mathrm{msec}$ ) before disappearing. The initial direction of movement of the stimulus was randomly either up or down the screen. With these oscillations occurring behind a prominent and continuously visible fixation point, the effect of eye movement was minimized. The stimulus reversed direction too frequently for it to be followed closely with saccadic eye movements (Kingstone \& Klein, 1993). Also, smooth pursuit is "totally ineffective" for the frequency and amplitude of the stimulus movement (Martins, Kowler, \& Palmer, 1985).

\section{Procedure}

In the main experiment, the inset and surround gratings together assumed one of nine spatial frequencies: $1.5,2.3,3.5,5.1,6.7,8.7$, $9.7,10.9$, and $14.5 \mathrm{cpd}$, for each of the following five $\theta \mathrm{s}: 22.5^{\circ}, 45^{\circ}$, $60^{\circ}, 90^{\circ}$, and $135^{\circ}$. The pixel size of our monitor did not permit us to adequately test spatial frequencies beyond $14.5 \mathrm{cpd}$. In case the strength of the relative motion illusion was dependent on the visibility of the stimulus, we first determined each observer's threshold contrast for each combination of spatial frequency and angle. A particular stimulus was subsequently presented at a high contrast constituting a constant multiple of the threshold contrast. This constant always exceeded 15 .

A method-of-adjustment procedure was used to measure threshold. The stimulus was presented in exactly the same manner as in the main experiment: rapidly oscillation lasting about a second. The observer adjusted the contrast of the stimulus just until a level was reached at which the grating structure of the stimulus could be resolved. A minimum of five threshold levels were averaged for each observer for most angle and frequency combinations, and thresholds for the other combinations were interpolated from these data. As an indication of the variation in visibility of the stimulus, Table 1 contains typical threshold contrast data.

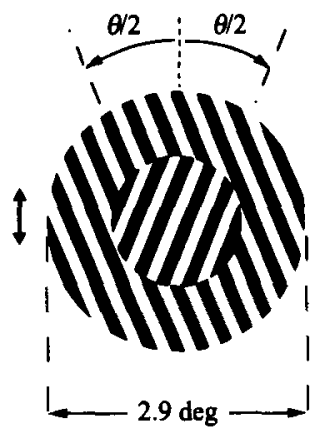

Figure 3. Schematic of the stimulus (to scale). Both the inset and surround were striped with 1-D sine-wave modulation (here represented as square waves) of achromatic contrast about the background level. The stimulus appeared on the monitor for about $1 \mathrm{sec}$, oscillating rapidly as indicated by the double-ended arrow on left. There was a fixation bead $\left(0.1^{\circ}\right.$ diameter) affixed to the screen over the center of the stimulus.
The subject's task was identical in all subsequent parts of all experiments. With his/her head fixed in a chin and forehead restraint, the subject viewed binocularly the fixation bead $\left(0.1^{\circ}\right.$ diameter $)$ that had been placed over the center of the stimulus (Figure 3 ). The subject pressed a button to start a trial. After each trial, he/she was required to press one of two buttons on a response box to indicate whether he/she had perceived any apparent sliding of the inset of the figure and/or stripes within the inset with respect to the surround, which was correlated with the oscillation of the whole figure. If such motion was perceived, the subject was required to press one of four buttons on the response box to indicate the strength of this apparent motion. Strength was determined primarily by the size of the apparent displacements of the inset produced by the oscillations of the entire stimulus.

In the main experiment, an experimental run consisted of eight stimulus presentations for each of the nine spatial frequencies for a fixed $\theta$, with the trials presented in a random order. Within each $\theta$, the gratings were presented at high contrast in such a way that the different spatial frequencies were matched for visibility. Data from at least two runs were collected for each and every $\theta$ totaling over 1,000 trials for each observer in this experiment. Data were gathered over a number of days.

In an ancillary experiment, we varied the contrast of a stimulus that had elicited a strong and reliable illusion in the first part of the experiment: $\theta=45^{\circ}$ at a spatial frequency of $9.7 \mathrm{cpd}$. A run consisted of eight trials at each of eight contrast levels: $4,6,8,10,12,16,24$, and $48 \times$ threshold, where order of presentation was randomized. Data from four runs conducted over at least 2 days were collected from Subject T.J.H. and data from three runs were collected from Subject G.T.R.

In another ancillary experiment, the spatial frequencies of both the inset and surround were independently varied and were chosen from the following values in 16 combinations: $14.5,9.7,3.2$, and $1.6 \mathrm{cpd}$, where $\theta$ was set at $45^{\circ}$ and all stimuli were presented at $38 \times$ threshold. A run consisted of six presentations of each of the 16 combinations with trial order completely randomized. Data from four runs occurring on different days were gathered from T.J.H. and G.T.R.

\section{RESULTS}

The percentage of trials on which the subject had perceived the illusion of relative motion of the inset was averaged for each angle and spatial frequency combination. Responses from the four buttons corresponding to the observer's rating of the strength of the illusion were scored as integers where 4 was the highest rating corresponding to the strongest illusion and 0 was assigned to trials in which no illusion was seen. These ratings were then averaged for each spatial frequency and angle combination; the results are plotted individually in Figures 4 and 5 .

Our two dependent measures of the illusion produced similar results, and a repeated measures analysis of variance (ANOVA) (spatial frequency $\times$ angle) was performed on each of the measures: percentage of trials on which the relative motion illusion was reported (\% relative motion) and the average rating of the strength of the illusion (average rating). There was a significant main effect for $\theta[\%$ relative motion, $F(4,12)=13.2, p<.025$; average rating, $F(4,12)=14.4, p<.025]$, for spatial frequency $[\%$ relative motion, $F(8,24)=12.9, p<.01$; average rating, $F(8,24)=8.0, p<.025]$, and for their interaction $[\%$ relative motion, $F(32,96)=4.7, p<.05$; average rating, $F(32,96)=5.2, p<.05]$. The main effect for angle could be accounted for by the fact that the illusion seemed 
Table 1

Threshold Contrast Values (in Percent) for Stimuli of Different Spatial Frequencies and Angles for Subject T.J.H.

\begin{tabular}{cccccc}
\hline \multirow{2}{*}{$\begin{array}{c}\text { Spatial } \\
\text { Frequency (cpd) }\end{array}$} & \multicolumn{5}{c}{ Angle $(\theta)$} \\
\cline { 2 - 6 } & $22.5^{\circ}$ & $45^{\circ}$ & $60^{\circ}$ & $90^{\circ}$ & $135^{\circ}$ \\
\hline 1.5 & 0.8 & 0.8 & 0.8 & 0.6 & 0.6 \\
2.3 & 0.4 & 0.4 & 0.7 & 0.6 & 0.5 \\
3.5 & 0.3 & 0.4 & 0.6 & 0.5 & 0.5 \\
5.1 & 0.4 & 0.5 & 0.6 & 0.6 & 0.7 \\
6.7 & 0.6 & 0.9 & 1.1 & 1.0 & 0.8 \\
8.7 & 1.0 & 0.8 & 1.7 & 1.2 & 1.2 \\
9.7 & 1.1 & 1.2 & 2.1 & 2.3 & 2.3 \\
10.9 & 1.3 & 1.6 & 2.6 & 2.4 & 2.4 \\
14.5 & 1.8 & 2.3 & 3.8 & 4.8 & 5.7 \\
\hline
\end{tabular}

to occur reliably only for quite acute $\theta$ s. We tested this hypothesis by performing post hoc means comparisons for the three $\theta$ s less than $90^{\circ}$ versus the other two angles. The acute angles produced a significantly greater illusory effect [ $\%$ relative motion, $F(1,12)=48.6, p<.005$; average rating, $F(1,12)=49.9, p<.005]$. The illusion worked well for spatial frequencies between 6 and $12 \mathrm{cpd}$, as indicated by post hoc comparisons conducted on these spatial frequencies, versus both the higher and lower spatial frequencies [\% relative motion, $F(1,24)=61.8, p<.002$; average rating, $F(1,24)=50.9, p<.002]$. In this respect, Subject L.V. showed the effect at spatial frequencies that were somewhat lower than those of the other 3 subjects. The illusion was almost always very weak for the highest spatial frequency of $14.5 \mathrm{cpd}$.
Figure 6 records the results from the first ancillary experiment, in which the contrast of a stimulus eliciting a strong illusion was varied. The illusion's strength saturates rapidly, showing some diminution at $4 \times$ threshold (contrast $\approx 4 \%$ ). Figure 7 plots the results from the final experiment. Post hoc means comparisons revealed that the strongest illusion occurred when both the inset and surround gratings were $9.7 \mathrm{cpd}$ [ $\%$ relative motion, $F(1,9)=$ $37.9, p<.05$; average rating, $F(1,9)=240.1, p<.02]$. The illusion diminished in strength if either the inset or surround gratings deviated from this spatial frequency.

\section{DISCUSSION}

Wade (1977) presented an extensive review of movement illusions and other distortions seen in stationary, repetitive fine-grain patterns. This includes "streaming," "scintillation," and "jazzing" effects, and some of these effects are due to small transient fluctuations in the refraction of light by the lens (Campbell \& Robson, 1958). These fluctuations cannot be responsible for the Ouchi illusion, for it has been seen through a pinhole and by an aphakic patient, thus eliminating astigmatic error as its source. Also, all the other illusions involve piecemeal and random effects seen largely at slightly eccentric locations in the visual field. In contrast, the present illusion involves the appearance of coherent and smooth relative motion without occlusion, occurring when the pattern is viewed centrally.
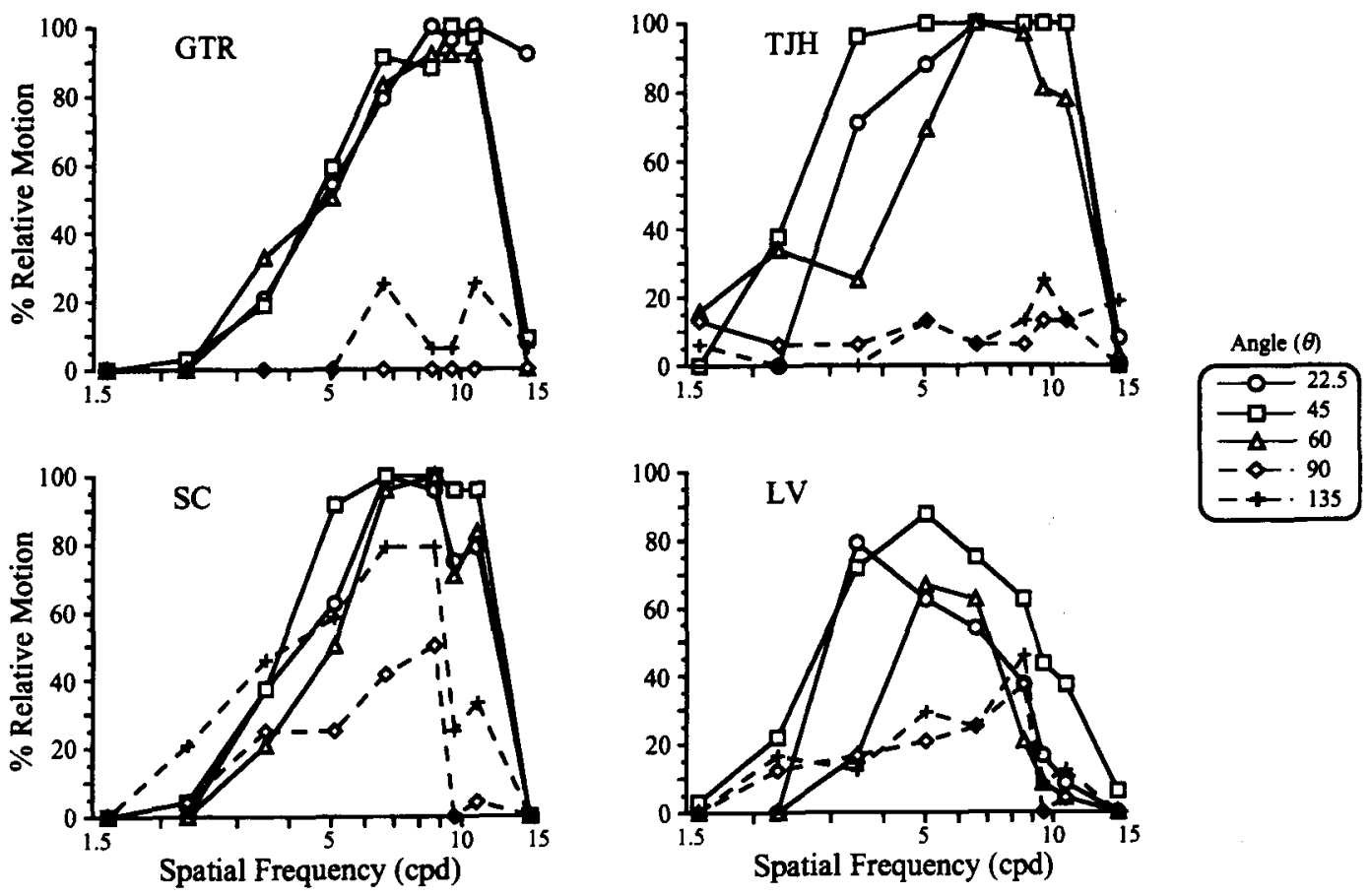

Figure 4. Frequency of reporting of the relative motion illusion as a function of the angle $\theta$ and spatial frequency of the stimulus, plotted for each subject. 

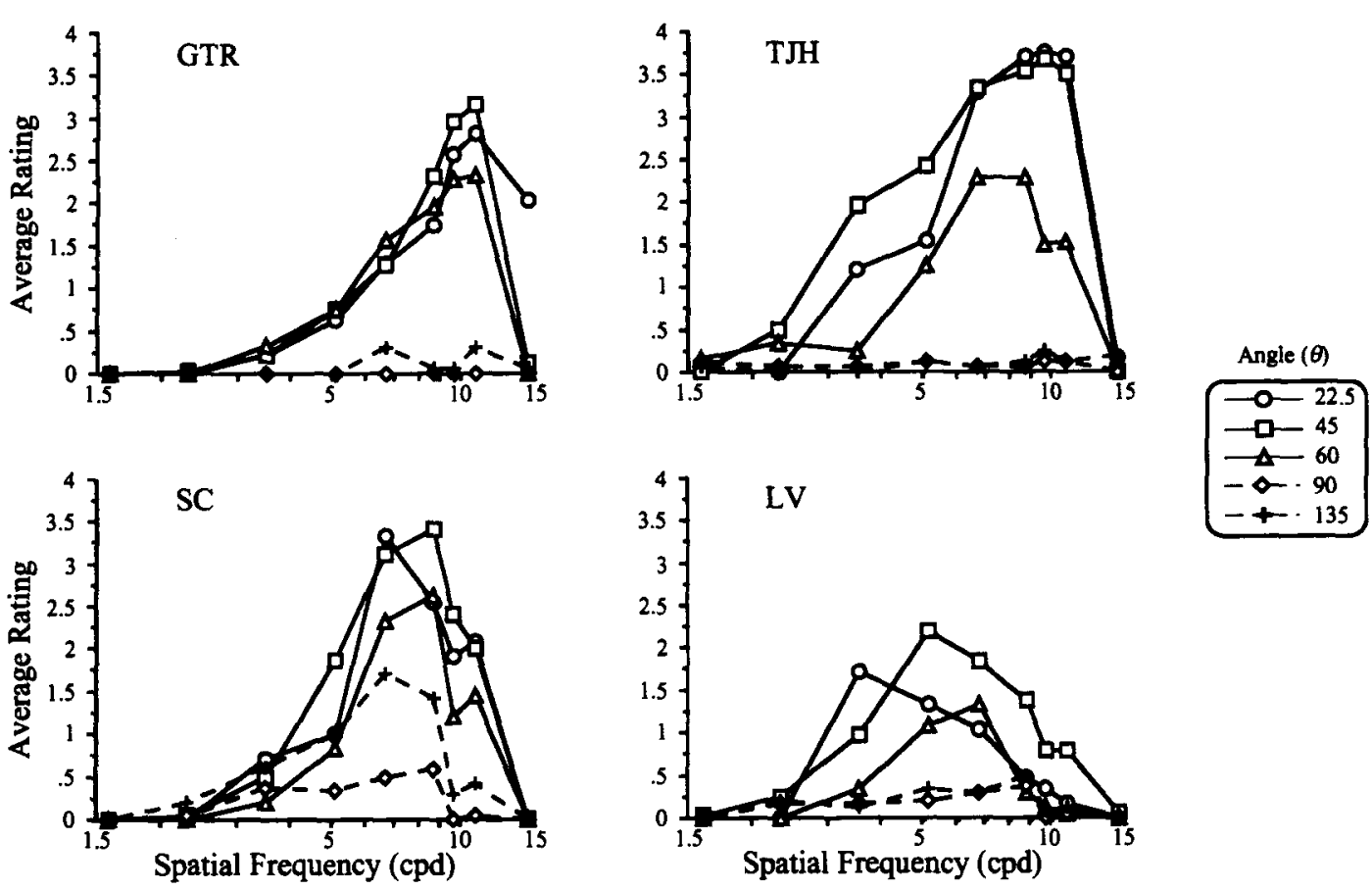

Figure 5. Average ratings of the illusion's strength as a function of the angle $\theta$ and spatial frequency of the stimulus, plotted for each subject.

The perception of relative motion in our simplified Ouchi pattern confirms that the illusion can be generated by rigid motion of a pattern containing elements of contrasting orientation. This provides circumstantial evidence in support of the suggestion that the motion in the original Ouchi pattern is due to the orientation contrast between the oblique harmonics of each component checkerboard texture. The reader can confirm our findings about orientation by viewing either of the stimuli in Figure 2 from the appropriate distance while tracking a small target (e.g., tip of a pencil) oscillated in front of the stimulus. The direction of tracking in relation to the orientation of the gratings is crucial. If it forms a sharp angle with the wavefronts of the component gratings, a strong illusion of sliding of the inset is produced. If the same direction of tracking is maintained, but the stimulus is rotated $90^{\circ}$ in the frontoparallel plane, making the angle between the tracking and the gratings more obtuse, the illusion weakens considerably.

Both Figures 1 and 2 generate a marked figure-ground effect of relative motion illusion in the manner of classically induced motion, where in this case, veridical motion of one pattern adjacent to another stationary pattern causes the latter to appear to move (Duncker, 1929/1938). In Figure 1 and in the upper stimulus in Figure 2, illusory movement is primarily seen in the "figure"-that is, the inset - as opposed to the "ground," or surround. Presumably the ground is stabilized with respect to an external, "absolute" frame of reference (e.g., the edges of the page or TV screen). What constitutes "figure" and "ground" is not as clear in the lower stimulus in Figure 2. Some- times the middle ring becomes the "figure" and hence it seems to move. On other occasions the central disk seems to move within the middle ring, or both the disk and middle ring move reciprocally within a stationary outer ring.

The illusion seen in the Ouchi figure, as well as our simplification, involves a response to local rather than global motion. It appears that the percept of relative motion in Figure 2 is determined entirely by the contrast between the local directions of motion orthogonal to the grating wavefronts, in spite of information indicating rigid motion that comes from two sources. The first is the identical movement of the bounding contours of the inset and surround. The second is the absence of deletion or accretion at the inset-surround boundary. It is unclear why global motion integration is failing in displays of this type. It is also unclear why the horizontal and vertical edges of the component rectangular checks in the original Ouchi pattern (Figure 1) do not provide a strong signal as to the veridical motion of the pattern as a whole. It is possible that motion information provided by these edges is being inhibited by the motion signal from the oblique harmonics between 6 and $12 \mathrm{cpd}$ of the pattern, which causes the illusion. Then again, as opposed to our simplification (Figure 2), the checkerboard texture of the Ouchi pattern seems to produce an illusion with a more pronounced low spatial frequency cut-off. Continuing the previous line of argument, it is possible that the local edges of the component rectangles of the Ouchi pattern are of sufficient salience to overcome the signal from the oblique harmonics and the illusion breaks down. An ana$\log$ of this scale dependence in an illusion has been found 

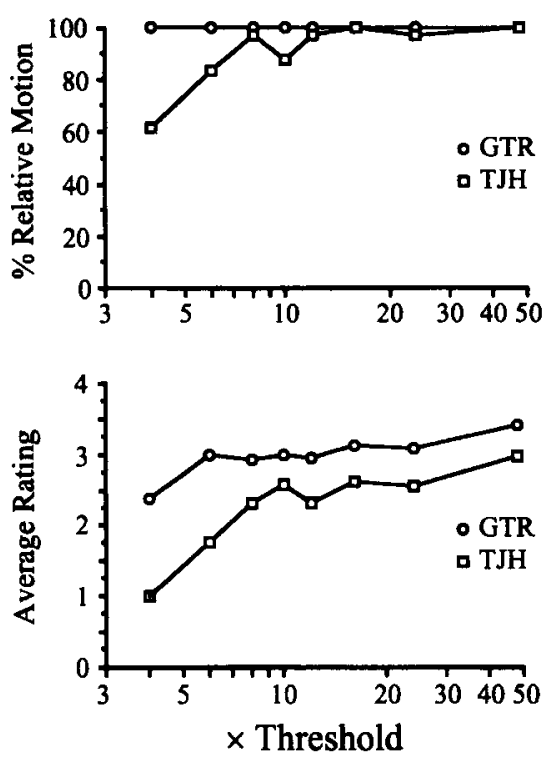

Figure 6. Effect of varying contrast for inset and surround gratings at 9.7 cpd and $\theta=45^{\circ}$. Upper graph shows the percentage of trials on which subjects (G.T.R. and T.J.H.) reported the illusion; lower graph shows the average ratings of the illusion's strength.

in the orientation-contingent color aftereffect of McCollough (Stromeyer, 1972).

Given that the Ouchi illusion exists because of an anomaly in the integration of local velocity signals into a rigid percept, what is the source of this anomaly? We conjecture that it is related to activity in grating cells, which have recently been discovered in macaque cortical areas V1 and V2 (von der Heydt, Peterhans, \& Dursteler, 1992). The narrow range of parameter values that we have found to characterize the illusion is very closely matched to the response of the grating cells, especially in terms of spatial frequency. Our computation of the overall spatial frequency response of the grating cells (thick solid line in Figure 8) was derived from these previous data (von der Heydt et al., 1992; see their Figure 5, pp. 1420-1421). Like us, these earlier workers used a $2 \mathrm{deg}^{2}$ stimulus presented centrally. Each cell is assumed to have a log-normal tuning curve, and the most common center frequency found was $8 \mathrm{cpd}$. The cells have a narrow spatial tuning curve, and guided by von der Heydt et al.'s data, we have assumed a constant half-height tuning width of 1.12 octaves. Also plotted in Figure 8 is the subjects' response to our stimulus for the most acute $\theta$ between inset and surround (Figure 3). Our reasons for using this particular angle are given below. The data of Subject L.V. are not included in Figure 8, since given her history of eye problems we assumed that her data were somewhat anomalous in that the peaks were shifted to lower spatial frequencies. Finally, in Figure 8 (thick dashed line), we have plotted (as a function of spatial frequency) the frequency of the reporting of smooth motion versus stationary flicker in suprathresh- old counterphase-modulated gratings corresponding to a drift velocity of $1.0 \% \mathrm{sec}$, which is near the velocity of our stimulus. The data are from Gorea and Lorenceau (1984). Once again, these data follow the spatial frequency response of the grating cells, providing circumstantial evidence that these cells are involved in the perception of smooth motion.

In addition to the spatial frequency match and paralleling the nature of the illusion itself, grating cells respond well near threshold contrast levels and are activated strongly by the retinal movement of an image of a stationary grating due to miniature fixational eye movements. Further, they are driven by checkerboard patterns similar to those in Figure 1, and they require a couple of cycles of a periodic pattern before they start responding above spontaneous activity levels. In case the spatial frequency effect revealed by the results in the main experiment (Figures 4 and 5) was being confounded with the number of periods in the stimulus, stimuli the same as those shown in Figure 3 were rendered on paper in very high contrast over a range of sizes. We informally noted that this spatial frequency effect was not dependent on the number of cycles in the gratings, provided that there was a minimum of about three cycles in the stimulus. The correspondence between our data and the behavior of the grating cells provides a prima facie case that these cells are implicated in the processing stream that extracts the motion seen in our display. Of course, this correlation alone does not necessarily establish a causal relation.

It has been found psychophysically that at least $120^{\circ}$ is required between directions of motion to stimulate entirely independent motion mechanisms (Ball, Sekuler, \& MacHamer, 1983). With grating cells having a much narrower orientation tuning than these motion mechanisms, the conclusion is that our illusion occurs when different motion channels and grating cells are being stimulated by inset and surround.

If grating cells are assumed to drive the illusion, what is the role of the angle between the two parts of our stimulus? There is a difference of $(180-\theta)^{\circ}$ between the inset and surround local motion vectors in Figure 3, so that acute angles between the gratings correspond to obtuse angles between the local motion vectors. Large angles between local motion vectors seem to be the cause of the effect reported by Nakayama and Silverman (1988), in which nonrigid, or undulating, motion is seen in a physically rigid wavy line of shallow amplitude as it is translated in the plane. Nakayama assumed that the computed velocity signal of a rigid object is obtained from the intersection of constraint lines defined by local velocity components, following Adelson and Movshon's (1982) explanation of coherent motion in plaids. With large angles between the local motion vectors and given noise in the resolution of both their direction and magnitude, the computed magnitude of the global velocity signal becomes very uncertain from moment to moment, and non-rigid motion is seen. Bearing this in mind, our smallest stimulus angle ensures 

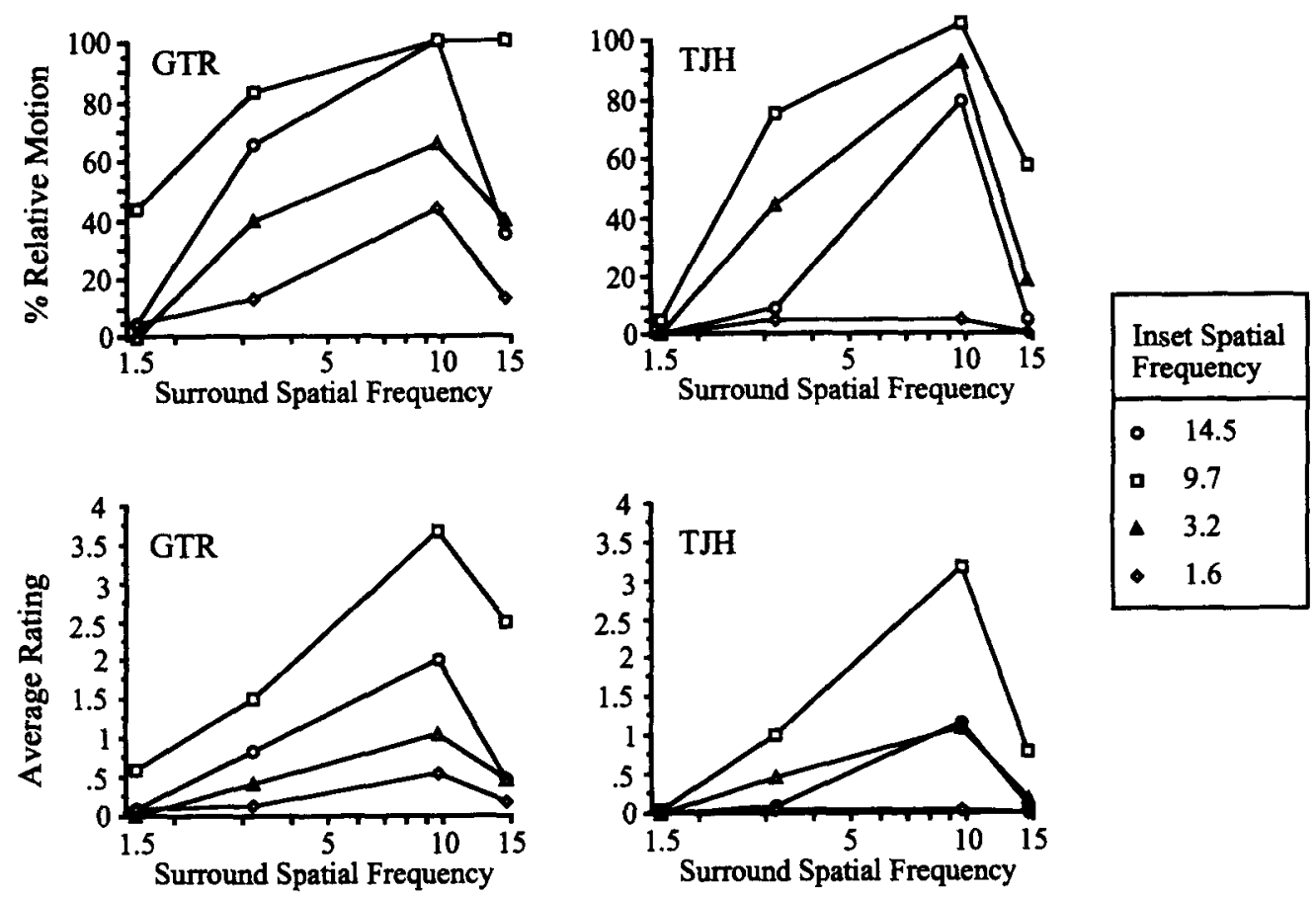

Figure 7. Effect of varying independently the spatial frequency (cpd) of the inset and surround for 2 subjects (G.T.R. and T.J.H.) where $\theta=45^{\circ}$. Upper graphs show the percentage of trials on which subjects reported the illusion; lower graphs record the average ratings of the illusion's strength.

the most conducive conditions for nonrigid motion to be seen between the inset and surround (Figure 8).

Finally, our data can be related to recent research on motion coherence in plaids. In data consistent with those

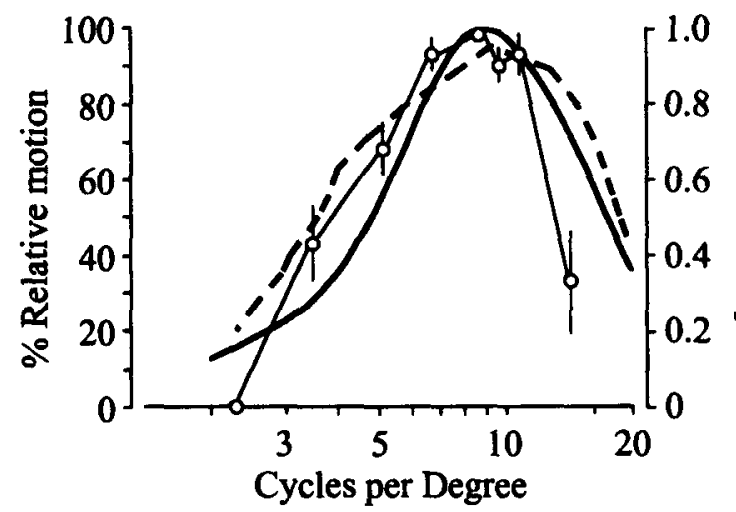

是

Figure 8. Relative spatial frequency response of grating cells in the primate visual cortex compared with the strength of the illusion. The open circles represent data ( $\%$ relative motion) from the main experiment averaged across Subjects G.T.R., T.J.H., and S.C. for $\theta=22.5^{\circ}$ and error bars are $\pm 1 S E M$. The thick solid line shows our computation of the response of all the grating cells recorded in areas $\mathrm{V} 1$ and $\mathrm{V} 2$ of macaque to sinusoidal gratings of high contrast (von der Heydt, Peterhans, \& Dursteler, 1992). The thick dashed line has been replotted from Gorea and Lorenceau (1984; Figure 5). In the latter data, vertical suprathreshold counterphase-modulated gratings of varying spatial frequencies generated in a $3.5^{\circ}$ window were seen either to drift smoothly left or right or to remain stationary and flicker. Further details are provided in the text. from the present study and from Nakayama and Silverman (1988), Kim and Wilson (1993) have shown that for obtuse angles between component vector directions, coherence in plaids disappears, to be replaced with "sliding" motion of the components. Also, our stimulus (Figure 2) can be interpreted as a "plaid" with no overlap between the components, and Kooi, de Valois, Switkes, and Grosof (1992) have shown that coherence rapidly disappears if there is only partial overlap between the component gratings.

\section{REFERENCES}

AdELSON, T., \& Movshon, J. A. (1982). Phenomenal coherence of moving visual patterns. Nature, 300, 523-525.

Ball, K., Sekuler, R., \& MacHamer, J. (1983). Detection and identification of moving targets. Vision Research, 23, 229-238.

Camprell, F. W., \& Robson, J. G. (1958). Moving visual images produced by regular stationary patterns. Nature, 181,362 .

DUNCKER, K. (1938). Induced motion. In W. D. Ellis (Ed.), A source book of Gestalt psychology (pp. 161-172). London: Routledge \& Kegan Paul. (Original work published in 1929)

Gorea, A., \& Lorenceau, J. (1984). Perceptual bistability with counterphase gratings. Vision Research, 24, 1321-1331.

Hine, T. J., COOK, M., \& Rogers, G. T. (1995). An illusion of relative motion dependent upon spatial frequency and orientation. Vision Research, 35, 3093-3102.

Khang, B., He, Z., \& Essock, E. A. (1995). Illusory motion and depth in the Ouchi pattern. Investigative Ophthalmology \& Visual Science, 36 (Suppl.), S362.

KiM, J., \& WiLSON, H. R. (1993) Dependence of plaid motion coherence on component grating directions. Vision Research, 33, 2479-2489.

Kingstone, A., \& KLEIN, R. M. (1993). What are human express saccades? Perception \& Psychophysics, 54, 260-273.

Kooi, F. L., de Valois, K. K., Switkes, E., \& Grosof, D. H. (1992). 
Higher-order factors influencing the perception of sliding and coherence of a plaid. Perception, 21, 583-598.

Martins, A. J., Kowler, E., \& PAlmer, C. (1985). Smooth pursuit of small-amplitude sinusoidal motion. Journal of the Optical Society of America A, 2, 234-242.

Nakayama, K., \& Silverman, G. H. (1988). The aperture problem I: Perception of nonrigidity and motion direction in translating sinusoidal lines. Vision Research, 28, 739-746.

OucHI, H. (1977). Japanese and geometrical art. New York: Dover.

Spillmann, L., Tulunay-Keesey, U., \& Olson, J. (1993). Apparent motion in normal and stabilized vision. Investigative Ophthalmology \& Visual Science, 34 (Suppl.), 1031.

SPILlMANN, L., \& WeRnER, J. S. (1990). Visual perception: The neurophysiological foundations. San Diego: Academic Press.
Steinman, R. M., Haddad, G. M., Skavenski, A. A., \& Wyman, D. (1973). Miniature eye movement. Science, 181, 810-819.

STROMEYER, C. F., III (1972). Edge-contingent color after effects: Spatial frequency specificity. Vision Research, 12, 717-733.

von der Heydt, R., Peterhans, E., \& Dursteler, M. R. (1992).

. Periodic-pattern-selective cells in monkey visual cortex. Journal of Neuroscience, 12, 1416-1434.

WADE, N. J. (1977). Distortions and disappearances of geometrical patterns. Perception, 6, 407-433.

(Manuscript received July 8, 1994; revision accepted for publication May 20, 1996.) 